\title{
Heat-shock-responsive genes are not involved in the adult diapause of Drosophila triauraria
}

\author{
Shin G. Goto and Masahito T. Kimura
}

\begin{tabular}{|c|l|}
\hline Citation & Gene. 326; 117-122 \\
\hline Issue Date & 2004-02-04 \\
\hline \multicolumn{1}{|c|}{ Type } & Journal Article \\
\hline Textversion & author \\
\hline & $\begin{array}{l}\text { C } 2003 \text { Elsevier B.V. All rights reserved. This manuscript version is made available } \\
\text { under the CC-BY-NC-ND 4.0 License . } \\
\text { https://creativecommons.org/licenses/by-nc-nd/4.0/. }\end{array}$ \\
Rights & $\begin{array}{l}\text { This is the accepted manuscript version. The article has been published in final form } \\
\text { at https://doi.org/10.1016/j.gene.2003.10.017. }\end{array}$ \\
& $\begin{array}{l}\text { Please cite only the published version. } \\
\text { 引用の際には出版社版をご確認ご利用ください。 }\end{array}$ \\
\hline DOI & \begin{tabular}{l}
$10.1016 /$ j.gene.2003.10.017 \\
\hline
\end{tabular}
\end{tabular}

Self-Archiving by Author(s)

Placed on: Osaka City University 


\title{
Heat-shock-responsive genes are not involved in the adult diapause of Drosophila triauraria
}

\author{
Shin G. Goto ${ }^{\mathrm{a}, *}$, Masahito T. Kimura ${ }^{\mathrm{b}}$ \\ ${ }^{a}$ Department of Bio- and Geosciences, Graduate School of Science, Osaka City University, Osaka 558-8585, Japan \\ ${ }^{\mathrm{b}}$ Graduate School of Environmental Earth Science, Hokkaido University, Sapporo 060-0810, Japan
}

\begin{abstract}
Although the molecular regulation of diapause remains largely unknown, there is an accumulation of data suggesting the involvement of heat-shock proteins in the expression of diapause or dormancy. However, Goto et al. [J. Insect Physiol. 44 (1998) 1009] reported that Drosophila triauraria does not express $H s p 70$ transcripts at normal temperatures, regardless of the diapause state. Here, we investigated the transcriptional regulation of other heat-shock-responsive genes (Hsp23,Hsp26,Hsp83 and Hsr $\omega)$ in D. triauraria with relation to diapause. The results revealed that these genes are not regulated as a function of diapause, suggesting that they are not involved in the expression of diapause in this species.
\end{abstract}

Keywords: Drosophila triauraria; Heat-shock-responsive genes; Diapause

\section{Introduction}

Diapausing individuals demonstrate striking differences in gene expression when compared with nondiapausing ones: i.e., most of genes are silenced but a select few are highly up-regulated during diapause (Denlinger, 2002). Some of the up-regulated genes encode heat-shock proteins (HSPs). HSPs are in a ubiquitous group of highly conserved proteins that are expressed in response to stress-induced protein denaturation. In nonstressed cells, one of their major functions is as molecular chaperones assisting in protein folding and in the assembly of protein complexes. In stressed cells, these proteins inhibit formation of protein aggregates, refold proteins or target proteins for degradation (Parsell and Lindquist, 1993; Hartl, 1996).

In the flesh fly Sarcophaga crassipalpis, some Hsps, Hsp23, Hsp70 and Hsp90, are transcriptionally regulated without any stresses when pupae enter diapause (Yocum et al., 1998; Rinehart and Denlinger, 2000; Rinehart et al., 2000). While the functions of HSPs during diapause remain unknown, such regulation has been observed not only in other diapausing insects but also in dormant or dauer organisms (reviewed by Denlinger, 2002). However, this is not the case in Drosophila triauraria. Goto et al. (1998) reported that $H s p 70$ transcripts are undetectable at normal temperatures, irrespective of the diapause state in this species.

In the light of above information, we investigated expressions of other heat-shock-responsive genes (Hsp23, Hsp26, Hsp83 and Hsr $\omega$ (Heat shock RNA $\omega)$ ) in diapausing individuals of D. triauraria. Hsp23 and Hsp26 belong to the small Hsp (smHsp) family and $H s p 83$ belongs to the $H s p 90$ family. Hsrc does not belong to the Hsps but is also responsive to heat shock as well as other stresses such as benzamide, colchicine and other amides. Unlike most of the widely studied genes encoding HSPs or other stress proteins, Hsrw transcripts do not code any protein (Lakhotia et al., 1999). D. triauraria is an excellent model system to strengthen our understanding of the relevance of the heat-shock-responsive genes in relation to diapause because its diapause has been extensively studied. This species occurs in subtropical and temperate areas of East Asia (Lemeunier et al., 1986; Kimura, 1987) and its temperate populations pass the winter by entering reproductive diapause in response to short daylength and low temperature in autumn (Kimura, 1983, 1984, 1988; Kimura et al., 1992). Previous studies revealed that diapausing individuals accumulate energy reserves and acquire tolerance to cold, heat and desiccation (Kimura, 1988; Ohtsu et al., 1992, 1993, 1998; Matsunaga et al., 1995; Goto et al., 1997, 1998). Daibo et al. (2001) reported that diapausing individuals of this species specifically express antifungal peptide genes, drosomycin and drosomycin-like, even in the absence of a microbial challenge. 
2. Materials and methods

\subsection{Experimental flies}

The strain of D. triauraria Bock and Wheeler originated from Onuma, northern Japan $\left(42^{\circ} \mathrm{N}\right)$, and was maintained for more than 10 years under diapause-preventing laboratory conditions (continuous light at $23{ }^{\circ} \mathrm{C}$ ) on cornmeal-malt medium. Flies were reared from the egg stage under diapause-inducing (10L14D, $10 \mathrm{~h}$ light-14 h dark) or diapause-averting (15L9D, $15 \mathrm{~h}$ light-9 h dark) photoperiods at $15^{\circ} \mathrm{C}$. In the present study, only males were used. Flies were frozen with liquid nitrogen and stored at $-80^{\circ} \mathrm{C}$.

\subsection{Heat shock}

Prior to experiments, vials that contained food medium were warmed to $37{ }^{\circ} \mathrm{C}$. Flies were introduced into these vials and exposed to heat for $30 \mathrm{~min}$.

\subsection{Molecular techniques}

Total RNA was extracted from the whole body using TRIzol (Invitrogen) according to the supplier's instruction. DNA was digested with DNase I (Roche). RNA was dissolved in RNase-free water and stored at $20^{\circ} \mathrm{C}$.

To investigate expression of $H_{s p 23}, H s p 26, H s p 83$ and $H s r \omega$ in D. triauraria, reverse transcription-polymerase chain reaction (RT-PCR) methodology was adopted. Expression of RpL32 (ribosomal protein L32) was also investigated as a control. RNAs were converted into cDNAs using random hexamer and M-MLV reverse transcriptase (Invitrogen) according to the supplier's instruction. The PCR reaction used the cDNA sample in a final concentration of $1 \mathrm{x}$ PCR buffer as formulated by Invitrogen, $0.2 \mu \mathrm{M}$ of each primer set shown in Table $1,0.1 \mathrm{mM}$ of dNTP, $1.5 \mathrm{mM}$ (3.0 mM for $H_{s} r \omega$ ) of $\mathrm{MgCl}_{2}$ and 0.025 $\mathrm{U} / \mu \mathrm{l}$ of Platinum Taq DNA polymerase (Invitrogen). Amplification was achieved with a preheat for 2 min at $94{ }^{\circ} \mathrm{C}, 28-32$ cycles of denaturing for $15 \mathrm{~s}$ at $94{ }^{\circ} \mathrm{C}$, annealing for $15 \mathrm{~s}$ at $60,58,48,56$ and $55^{\circ} \mathrm{C}$ for Hsp83, Hsp26, Hsp23, Hsrc and $R p L 32$, respectively, and extension for $30 \mathrm{~s}$ at $72{ }^{\circ} \mathrm{C}$, and a final extension of $72{ }^{\circ} \mathrm{C}$ for $7 \mathrm{~min}$. The PCR products were separated by electrophoresis on agarose gel and DNA bands were detected with ethidium bromide or SYBR green (Molecular Probes). In this experiment, PCR reactions with 28, 32, 36 and 40 cycles were performed, and it was confirmed that less than 32 cycles could avoid the plateau effects.

To verify that the primers listed in Table 1 could amplify the corresponding genes, amplified fragments were sequenced. The fragments were subcloned into a pGEM-T vector and transformed into competent cells according to standard protocol (pGEM-T vector system, Promega). Sequencing was performed on a Long-Read Tower DNA Sequencer with a Dual CyDye terminator sequencing kit (Amersham Biosciences). Sequences for Hsp23, Hsp26, Hsp83 and Hsr $\omega$ are deposited on DDBJ/GenBank/EMBL as the accession numbers AB111906, AB111907, AB111908 and AB111909, respectively.

In D. melanogaster, Hsr $\omega$ produces two primary nuclear transcripts. The first nucleus limited Hsr $\omega$-n transcript is about 10-20 kb long and spans the entire transcription unit. The second smaller transcripts, the Hsr $\omega$-pre-c, includes the two exons (exons 1 and 2) and a single intron. The intron in the transcript is spliced out to generate the cytoplasmic transcript, $H s r \omega$-c. The amplified fragment from $D$. triauraria in the present study corresponds to exon 2 that is included in all of the transcripts in D. melanogaster. Thus, the present study is expected to measure all of the Hsr $\omega$ transcripts in D. triauraria.

\section{Results}

\subsection{Sequences of Hsp23, Hsp26, Hsp83 and Hsrc}

Amplification of cDNA from $D$. triauraria using each primer set resulted in a single band. The amplified fragments were sequenced and 303, 372, 360 and 204 bp for Hsp23, Hsp26, Hsp83 and $H s r \omega$, respectively, were determined. Deduced amino acid sequences of $H s p 23$ and Hsp 26 from D. triauraria are highly conserved when compared with those from D. melanogaster (Fig. 1A,B). The HSP20 domain that is observed in the members of the smHSP family was detected in the sequences. In addition, D. triauraria Hsp83 was almost identical to others known from insects (Fig. 1C). On the other hand, the present study demonstrated that nucleotide sequences of Hsrw are poorly conserved, even among Drosophila species (Fig. 1D). 


\subsection{RT-PCR analyses}

While expression of $H s p 23, H s p 26$ and $H s r \omega$ was clearly induced by heat shock ( $37{ }^{\circ} \mathrm{C}$ for $30 \mathrm{~min}$ ), up-regulation of $H s p 83$ transcripts after heat shock was not apparent (Fig. 2). It is noteworthy that the expression of neither Hsp23, Hsp26, Hsp83 nor $H s r \omega$ was transcriptionally regulated as a function of diapause (Fig. 2): i.e., Hsp23 and Hsp26 transcripts were undetectable, while $H s p 83$ and $H s r \omega$ were transcribed at a moderate level, irrespective of the diapause state, at normal temperatures.

\section{Discussion}

Although members of the smHSP family are less conserved when compared with those of other HSP families (reviewed by Denlinger et al., 2001), amino acid sequences of HSP23 and HSP26 in D. triauraria are highly conserved when compared with those in D. melanogaster. Gupta (1995) detected five highly conserved regions in HSP90 amino acid sequences of known eukaryotes. Although a region that was amplified from D. triauraria $H s p 83$ in the present study does not correspond to the conserved region, amino acid sequences of the sequenced region are quite similar among known insects. Sequences of $H s r \omega$, on the contrary, are poorly conserved among Drosophila species as reported by Lakhotia et al. (1999).

All of the genes investigated in the present study are clearly responsive to severe heat shock $\left(37^{\circ} \mathrm{C}\right.$ for $\left.30 \mathrm{~min}\right)$ except Hsp83: i.e., up-regulation of $H s p 83$ transcripts is not apparent. This is consistent with the expression patterns in $D$. melanogaster. Lindquist (1980) reported that Hsp83 in D. melanogaster is induced by rather moderate heat shock when compared with other $H s p$ s, and its expression is suppressed by severe heat shock.

Expression of $H s p$ s during diapause has been extensively studied in the flesh fly $S$. crassipalpis. Hsp 23 and $H s p 70$ transcripts are highly up-regulated immediately upon entry into pupal diapause even in the absence of thermal stress (Yocum et al., 1998; Rinehart et al., 2000). Expression persists throughout diapause but declines within a few hours after diapause is terminated by an application of hexane. Such up-regulation has been observed in other dormant organisms: i.e., $p 26,26 \mathrm{kDa}$ smHsp in diapausing encysts of the brine shrimp Artemia franciscana, Hsp26 in dormant yeast Saccharomyces cerevisiae, Hsp 70 in dauer larvae of the nematode Caenorhabditis elegans and grp78, mitochondrial Hsp 70, in the ground squirrel Spermophilus tridecemlineatus (reviewed by Denlinger et al., 2001). In S. crassipalpis, Hsp 90 transcripts are down-regulated during diapause but are responsive to temperature stresses, and its transcripts are restored to prediapause levels within a few hours after the termination of diapause (Rinehart and Denlinger, 2000). Such transcriptional regulation in association with dormancy was also observed in S. cerevisiae and C. elegans (reviewed by Denlinger et al., 2001).

Thus, accumulated data suggest the involvement of $H_{s p}$ in the dormancies of organisms ranging from yeast to mammal. Although it is still unclear precisely what their functions may be, Denlinger et al. (2001) has hypothesized that HSPs act as chaperone molecules to maintain structural integrity of proteins during dormancy or possibly they directly contribute to the arrest of development.

In D. triauraria, however, smHsps (Hsp23 and Hsp26, the present study) and Hsp70 (Goto et al., 1998) transcripts are undetectable at normal temperatures, irrespective of the diapause state. In addition, no difference in $H s p 83$ expression is detected between diapausing and nondiapausing individuals of D. triauraria. Although there was no prior information on Hsr $\omega$ expression during diapause in any species, the present study revealed that $H s r \omega$ expression is not affected by the diapause state of this species. Thus, none of the heat-shock-responsive genes are regulated as a function of diapause, suggesting that they are not involved in the expression of diapause in D. triauraria. In insects, regulation of HSPs in response to diapause has been studied only in four species, D. triauraria (Goto et al., 1998; the present study), S. crassipalpis (Yocum et al., 1998; Rinehart et al., 2000; Rinehart and Denlinger, 2000), gypsy moth Lymantria dispar (Yocum et al., 1991; Denlinger et al., 1992) and Colorado potato beetle Leptinotarsa decemlineata (Yocum, 2001). The last two species also represent different responses from S. crassipalpis at least for the regulation of Hsp70. HSP70 is not expressed upon the initiation of diapause but only after cold temperature has been realized in Ly. dispar (Yocum et al., 1991; Denlinger et al., 1992). On the other hand, in Le. decemlineata, $L d H s p 70 A$, one of the two copies of $H s p 70$ gene, is slightly up-regulated during diapause but the intensity of the response is much weaker than observed in flesh flies, while $L d H S P 70 B$, the other, is completely undetectable (Yocum, 2001). Difference between the species showing up-regulation of $H s p 70$ during diapause (S. crassipalpis) and ones showing no or quite weak upregulation (D. triauraria, Ly. dispar and Le. decemlineata) might be dependent on the difference in the mode of cold-tolerance acquisition during diapause. In S. crassipalpis, cold tolerance is firmly linked to the expression of diapause and diapausing individuals are much more tolerant to severe cold than nondiapausing ones (Lee et al., 1987). On the other hand, in D. triauraria, 
the acquisition of cold tolerance during diapause was not so pronounced: i.e., a half lethal temperature was just $1{ }^{\circ} \mathrm{C}$ lower in diapausing individuals than in nondiapausing ones of D. triauraria (Goto et al., 1998). Diapause in adult Le. decemlineata induces a modest cold hardening via partial dehydration and elimination of endogenous gut nucleators (Costanzo et al., 1997). It is noteworthy that, in diapausing pharate larvae of $L y$. dispar, the capacity to synthesize HSPs is enhanced once the pharate larvae have experienced a period of chilling and the increased capacity elicited by chilling also coincides with an increase in cold tolerance (Yocum et al., 1991; Denlinger et al., 1992). A more extensive survey must be undertaken to determine how widespread the association between the HSP response and insect diapause is.

\section{Acknowledgements}

We are very grateful to anonymous reviewers for their invaluable suggestions and to Dr. H. Numata for his critical reading of this manuscript.

\section{References}

Bateman, A., Birney, E., Cerruti, L., Durbin, R., Etwiller, L., Eddy, S.R., Griffiths-Jones, S., Howe, K.L., Marshall, M., Sonnhammer, E.L., 2002. The Pfam protein families database. Nucleic Acids Res. 30, 276-280.

Costanzo, J.P., Moore, J.B. , Lee Jr., R.E., Kaufman, P.E., Wyman, J.A. 1997. Influence of soil hydric parameters on the winter cold hardiness of a burrowing beetle, Leptinotarsa decemlineata (Say). J. Comp. Physiol. B 167, 169-176.

Daibo, S., Kimura, M.T., Goto, S.G., 2001. Upregulation of genes belonging to the drosomycin family in diapausing adults of Drosophila triauraria. Gene $278,177-184$

Denlinger, D.L., 2002. Regulation of diapause. Annu. Rev. Entomol. 47, 93-122.

Denlinger, D.L., Lee Jr., R.E., Yocum, G.D., Kukal, O., 1992. Role of chilling in the acquisition of cold tolerance and the capacitation to express stress proteins in diapausing pharate larvae of the gypsy moth, Lymantria dispar. Arch. Insect Biochem. Physiol. 21, 271-280.

Denlinger, D.L., Rinehart, J.P., Yocum, G.D., 2001. Stress proteins: a role in insect diapause? In: Denlinger, D.L., Giebultowicz, J., Saunders, D.S. (Eds.), Insect Timing: Circadian Rhythmicity to Seasonality. Elsevier, Amsterdam, pp. 155-171.

Goto, S.G., Tagawa, M., Kimura, M.T., 1997. The effect of age, sex and diapause on desiccation tolerance in Drosophila triauraria (Diptera, Drosophilidae). Jpn. J. Entomol. 65, 362-368.

Goto, S.G., Yoshida, K.M., Kimura, M.T., 1998. Accumulation of Hsp70 mRNA under environmental stresses in diapausing and nondiapausing adults of Drosophila triauraria. J. Insect Physiol. 44, 1009-1015.

Gupta, R.S., 1995. Phylogenetic analysis of the $90 \mathrm{kDa}$ heat-shock family of protein sequences and an examination of the relationship among animals, plants, and fungi species. Mol. Biol. Evol. 12, 1063-1073. Hartl, F.U., 1996. Molecular chaperones in cellular protein folding. Nature 381, 571-580.

Kimura, M.T., 1983. Geographic variation and genetic aspects of reproductive diapause in Drosophila triauraria and Drosophila quadraria. Physiol. Entomol. 8, 181-186.

Kimura, M.T., 1984. Geographic variation of reproductive diapause in the Drosophila auraria complex (Diptera: Drosophilidae). Physiol. Entomol. 9, 425431.

Kimura, M.T., 1987. Habitat differentiation and speciation in the Drosophila auraria species-complex (Diptera Drosophilidae). Kontyû, Tokyo 55, 429-436.

Kimura, M.T., 1988. Interspecific and geographic variation of diapause intensity and seasonal adaptation in the Drosophila auraria species complex (Diptera: Drosophilidae). Funct. Ecol. 2, 177-183.

Kimura, M.T., Awasaki, T., Ohtsu, T., Shimada, K., 1992. Seasonal changes in glycogen and trehalose content in relation to winter survival of four temperate species of Drosophila. J. Insect Physiol. 38, 871-875.

Lakhotia, S.C., Ray, P., Rajendra, T.K., Prasanth, K.V., 1999. The noncoding transcripts of hsr-omega gene in Drosophila: do they regulate trafficking and availability of nuclear RNA-processing factors? Curr. Sci. 77, 553-563.

Lee Jr., R.E., Chen, C.-P., Meacham, M.H., Denlinger, D.L., 1987. Ontogenetic patterns of cold-hardiness and glycerol production in Sarcophaga crassipalpis. J. Insect Physiol. 33, 587-592.

Lemeunier, F., David, J.R., Tsacas, L., Ashburner, M., 1986. The melanogaster species group. In: Ashburner, M., Carson, H.L., Thompson Jr., J.N. (Eds.), The Genetics and Biology of Drosophila, vol. 3e. Academic Press, London, pp. 147-256.

Lindquist, S., 1980. Varying patterns of protein synthesis in Drosophila during heat shock: implications for regulation. Dev. Biol. 77, 463-479.

Matsunaga, K., Takahashi, H., Yoshida, T., Kimura, M.T., 1995. Feeding, reproductive and locomotor activities in diapausing and non-diapausing adults of Drosophila. Ecol. Res. 10, 87-93.

Ohtsu, T., Kimura, M.T., Hori, S.H., 1992. Energy storage during reproductive diapause in the Drosophila melanogaster species group. J. Comp. Physiol., B $162,203-208$.

Ohtsu, T., Katagiri, C., Kimura, M.T., Hori, S.H., 1993. Cold adaptations in Drosophila. Qualitative changes of triacylglycerols with relation to overwintering. J. Biol. Chem. 268, 1830-1834.

Ohtsu, T., Kimura, M.T., Katagiri, C., 1998. How Drosophila species acquire cold tolerance. Qualitative changes of phospholipids. Eur. J. Biochem. 252, 608611. 
Parsell, D.A., Lindquist, S., 1993. The function of heat-shock proteins in stress tolerance: degradation and reactivationof damaged proteins. Annu. Rev. Genet. 27, 437-496.

Rinehart, J.P., Denlinger, D.L., 2000. Heat-shock protein 90 is down-regulated during pupal diapause in the flesh fly, Sarcophaga crassipalpis, but remains responsive to thermal stress. Insect Mol. Biol. 9, 641-645.

Rinehart, J.P., Yocum, G.D., Denlinger, D.L., 2000. Developmental upregulation of inducible hsp70 transcript, but not the cognate form, during pupal diapause in the flesh fly, Sarcophaga crassipalpis. Insect Biochem. Mol. Biol. 30, 515-521.

Yocum, G.D., 2001. Differential expression of two HSP70 transcripts in response to cold shock, thermoperiod, and adult diapause in the Colorado potato beetle. J. Insect Physiol. 47, 1139-1145.

Yocum, G.D., Joplin, K.H., Denlinger, D.L., 1991. Expression of heat shock proteins in response to high and low temperature extremes in diapausing pharate larvae of the gypsy moth, Lymantria dispar. Arch. Insect Biochem. Physiol. 18, 239-249.

Yocum, G.D., Joplin, K.H., Denlinger, D.L., 1998. Upregulation of a $23 \mathrm{kDa}$ small heat shock protein transcript during pupal diapause in the flesh fly, Sarcophaga crassipalpis. Insect Biochem. Mol. Biol. 28, 677-682. 
Table 1

Sequences of primers

Oligonucleotides Sequence (5’>3')

\begin{tabular}{cccccccc}
\hline $\begin{array}{c}\text { For Hsp23 } \\
\text { Hsp23-F1 }\end{array}$ & CCA & YTR & TTG & TTG & AGC & CTT & \\
Hsp23-R1 & TAR & CGG & CGK & ACA & AAG & TGA & CG \\
For Hsp26 & & & & & & & \\
Hsp26-F1 & CTC & TGC & TTT & CGC & TTG & TGG & AT \\
Hsp26-R1 & TAG & CCA & TCG & GGA & ACC & TTG & TA \\
For Hsp83 & & & & & & & \\
Hsp83-F1 & AAG & GAG & TAC & AAG & GGC & AAG & CA \\
Hsp83-R1 & ACA & GCA & GGA & TGA & CCA & GAT & CC \\
For Hsr & & & & & & & \\
Hsr-F2 & TTG & GGC & GTT & GAA & AGT & TGA & TA \\
Hsr-R1 & CAA & TCC & GCA & CAA & TCA & ATC & TG \\
For RpL32 & & & & & & & \\
RPL49L-F1 & CAC & CAG & TCG & GAT & CGN & TAT & GCC \\
RPL49L-R1 & GAC & AGC & TGC & TTG & GCN & CGN & TC \\
\hline
\end{tabular}



A) $H s p 23$
D.triauraria
D.melanogaster

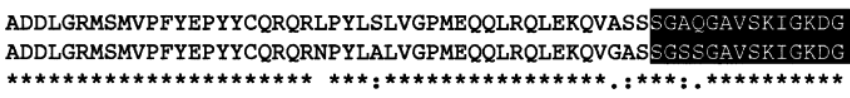
60

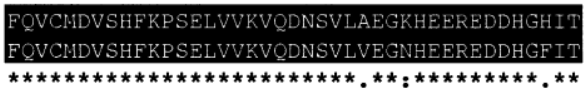
101

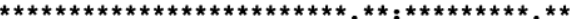
111
B) $\mathrm{Hsp} 26$
D.triauraria
ELQEPRSPMYEFSLGMHPHSRLLYPGVTSHRRSINGCPCASPICPASPAGQVMALRREMS
D.melanogaster

\section{C) Hsp83}

D.triauraria

D.auraria

D.melanogaster

A.albimanus LVSVTKEGLELPEDDAEKKKREEDKAKFESLCKLMNAILDNKVEKVVVSNRLVDSPCCIV LVSVTKEGLELPEDESEKKKREEDKAKFESLCKLMKSILDNKVEKVVVSNRLVDSPCCIV LVCVTKEGLELPEDEAEKKKREEDKAKFENLCKVMKSVLESKVEKVVVSNRLVDSPCCIV LVSVTKEGLELPEDEEEKKKREEDKVKFEGLCKVMKNILDNKVEKVVVSNRLVESPCCIV

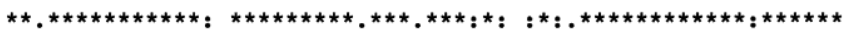

B.mori

TSQFGWSANMERIMKAQALRDTATMGYMAGKKQLEINPDHPIVETLRQKADADKNDKAV TSQFGWSANMERIMKAQALRDTATMGYMAGKRQLEINPDHPIVETLRQRADADKNDKAV TSQFGWSANMERIMKAQALRDTATMGYMAGKKQLEINPDHPIVETLRQKADADKNDKAV TSQYGWSANMERIMKAQALRDSSAMGYMAGKKHLEINPDHAIIETLRQRAEADKNDKAV TAQYGWSANMERIMKAQALRDTSTMGYMAAKKHLEINPDHSIVETLRQKAEADKNDKAV

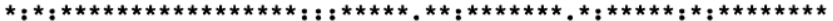

D) Hsrw

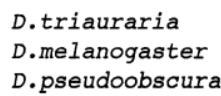

CGCGAAC---GTGAAA------ACTCAATACCCTGCGCGAGCCTGGGGCGGCATATGGGT ATCGATCC--GTGAAA------AGTCGATACCCTGCGCAAGCATGGGGCGGCATATGGGT ATCGATCCAAGTAAAACTCTGAACTCTGTACCCTTCGCGAGCAATAGGCCAGA-AGAAGT

GCTGAAAATGCACTCCGCCCCGTGCCCCAGAGCTCCTGCGTTTGGTCAGGGCTGCGTCTG 111 GCTGAAAACGCACTCGGCCC---GATCCCGAT-TGCAGCGTTATTCGAAAGCTGTGTCTG 1890 GCTGAAAATGCACTTCGGCCCATGTACGCGTT----TGGGCTACGCGAAAGTTGTGGTG 1721

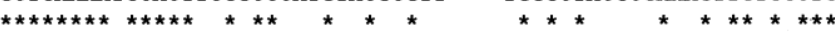

CGACTGTGACCGTGACTGAGATAACGCCCTGTGCGGTGTATCCGGGTTCTCGTGGTCGCG 171 CGA------CCGTGACTGAGATCATATGC-GTACA-TATATCTAATGTC-CGGGGTCGTG 1941 CGA------CCСTGACTCAG-------CCTTAGTG-TTGATT----GTC--GGGGTCGCG 1761

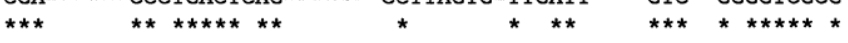

GGCCAGCCCAGCC-CG-GGGTGCTCGC-GATTCAGT 204 GGCCAGCCAGGGTGCT-CGATTCTGTCAGATTGATT 1976 GACTGACCAGGGTGCGTCGTTTCTATCAGATTGATT 1797

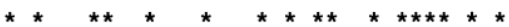

Fig. 1. Alignment of HSP23 amino acid sequences from D. triauraria and D. melanogaster (DDBJ/GenBank/EMBL accession numbers: AB111906 and J01100, respectively) (A), that of HSP26 ones from D. triauraria and D. melanogaster (DDBJ/GenBank/EMBL accession numbers: AB111907 and J01099, respectively) (B), that of HSP83 ones from D. triauraria, D. auraria, D. melanogaster, Anopheles albimanus and Bombyx mori (DDBJ/GenBank/EMBL accession numbers: AB111908, U75687, AE003477, L47285 and AB060275, respectively) (C), and that of Hsr $\omega$ nucleotide sequences from D. triauraria, D. melanogaster and D. pseudoobscura (DDBJ/GenBank/EMBL accession numbers: AB111909, U18307 and X16337, respectively) (D). '*': same residue (for Hsrw, same nucleotide), ':': strong positive residue, ' $:$ : weaker positive residue. Numbers on the right side are given according to each accession number. HSP20 domains detected by Pfam database (Bateman et al., 2002) were indicated by closed boxes. 
A)
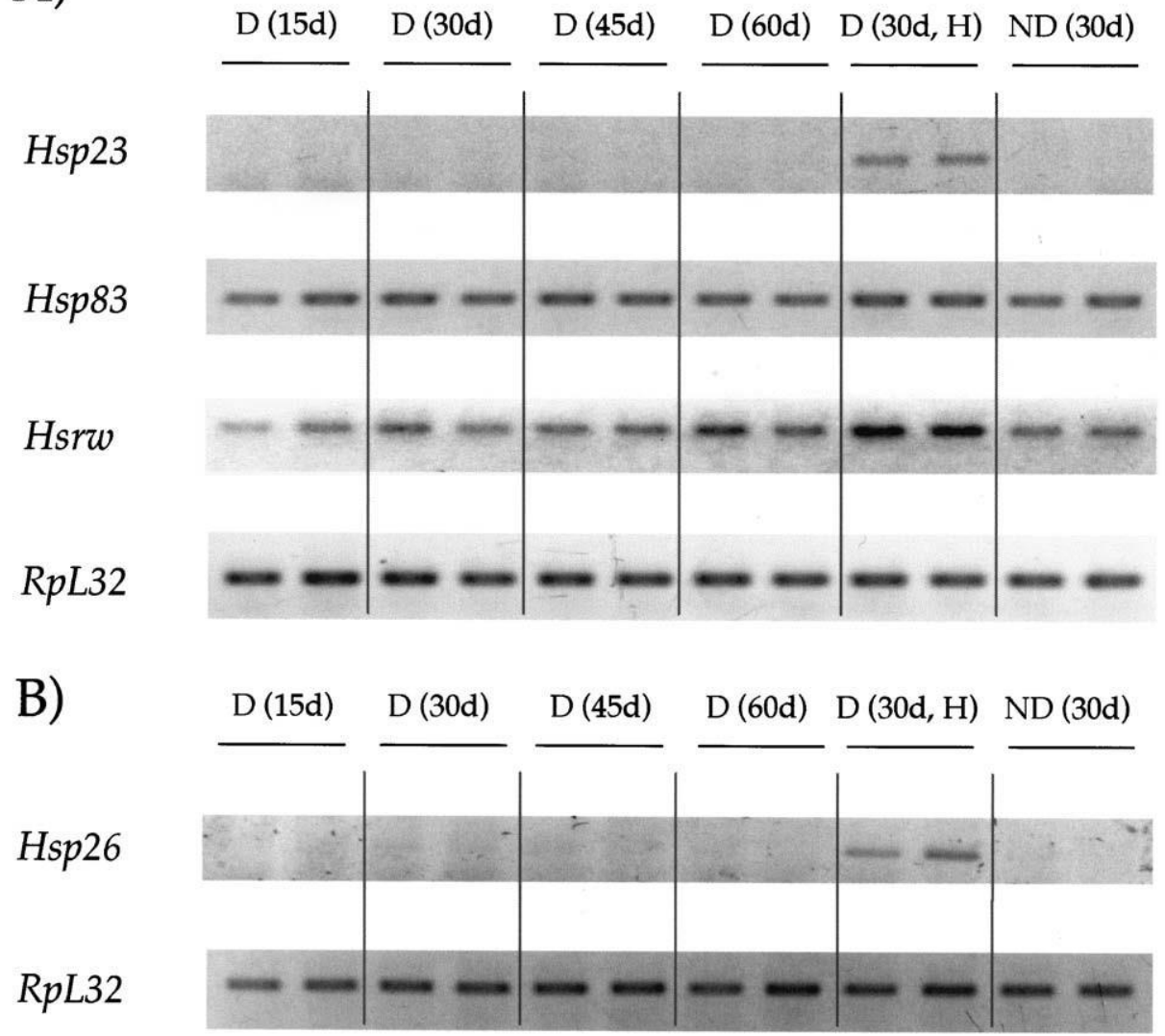

Fig. 2. Expression patterns of $H s p 23, H s p 83$ and $H s r \omega(\mathrm{A})$ and those of $H s p 26$ (B) in D. triauraria. Numbers in parentheses indicate the days after eclosion. $\mathrm{ND}$ : nondiapause, D: diapause, $\mathrm{H}$ : heat shock $\left(37^{\circ} \mathrm{C}, 30 \mathrm{~min}\right)$. 